\title{
Safety and effectiveness of reoperation for persistent or recurrent drug refractory secondary hyperparathyroidism
}

\author{
Lei Zhu, Feng Cheng, Xi Zhu, Bin Zhou, Yonghong Xu, Yong Wu, Chuxiao Shao \\ Department of Thyroid and Breast Surgery, The Fifth Affiliated Hospital of Wenzhou Medical University \& Lishui Hospital of Zhejiang University \\ \& Lishui Central Hospital, Lishui 323000, China \\ Contributions: (I) Conception and design: L Zhu, F Cheng, C Shao; (II) Administrative support: B Zou, Y Xu; (III) Provision of study materials: X \\ Zhu, B Zhou; (IV) Collection and assembly of data: X Zhu, B Zhou, Y Xu, Y Wu; (V) Data analysis and interpretation: L Zhu, F Cheng, C Shao; (VI) \\ Manuscript writing: All authors; (VII) Final approval of manuscript: All authors. \\ Correspondence to: Chuxiao Shao, MD. Department of Thyroid and Breast Surgery, The Fifth Affiliated Hospital of Wenzhou Medical University \& \\ Lishui Hospital of Zhejiang University \& Lishui Central Hospital, No. 289, Kuocang Road, Lishui 323000, China. Email: scx1818@126.com.
}

Background: Drug-refractory secondary hyperparathyroidism (SHPT) is the most common complication
in patients with chronic renal failure (CRF). Although surgery is the most effective and safe method for
drug-refractory SHPT, the condition may persist or recur after the primary surgery, and reoperation is often
required in these patients. The purpose of our current study was to evaluate the safety and effectiveness of
reoperation for drug-refractory SHPT.

Methods: The clinical data of 15 patients requiring reoperation after a surgery for drug-refractory SHPT in our hospital from 2010 to 2019 were retrospectively analyzed. Changes in biochemical markers including intact parathyroid hormone (iPTH), blood calcium (Ca), blood phosphorus (P), alkaline phosphatase (ALP), and blood calcium phosphorus product $\left(\mathrm{Ca}^{*} \mathrm{P}\right)$ were compared before and after the surgery, and the effectiveness and complications of the reoperation were summarized.

Results: The reoperation was successful in all the 15 patients after a single attempt. Routine pathological examinations identified a total of 25 parathyroid glands, of which 10 were in the neck in situ, 5 were ectopic in the neck, and 10 were in the forearm. The ectopic parathyroid glands were located inside the thyroid gland $(n=1)$, anterior superior mediastinum $(n=1)$, or thymus $(n=3)$. Surgical treatment significantly improved clinical symptoms such as skin pruritus and bone pain. Blood iPTH, Ca, P, ALP, and Ca*P were significantly reduced $(\mathrm{P}<0.05$ or $\mathrm{P}<0.01)$ after surgery. Hypothyroidism occurred in 1 patient; 4 patients undergoing orthotopic neck surgery developed transient hoarseness, which were alleviated within 6 months; no severe complications such as bleeding or death were noted. No recurrence occurred during the 6-month follow-up. Conclusions: Reoperation is safe and effective for drug-refractory SHPT. Preoperative imaging should be performed to achieve accurate positioning, and the recurrent laryngeal nerve should be closely monitored during surgery. The purpose of the reoperation is to remove all possible parathyroid tissues to avoid recurrence.

Keywords: Drug-refractory; secondary hyperparathyroidism (SHPT); parathyroidectomy; reoperation

Submitted Feb 07, 2020. Accepted for publication Apr 16, 2020.

doi: $10.21037 /$ gs-20-391

View this article at: http://dx.doi.org/10.21037/gs-20-391

\section{Introduction}

Secondary hyperparathyroidism (SHPT) after chronic renal failure (CRF) is predominantly caused by the development of hyperphosphatemia and vitamin D deficiency and resistance (1). Although SHPT can be controlled by medical therapies in most cases, it may progress into drug-refractory types. The SHPT patients who still show increased blood intact parathyroid hormone (iPHT) and uncontrollably high blood calcium and blood phosphorus levels after 
the use of drugs such as active vitamin $\mathrm{D}$ and calciumlike agents can be considered drug-resistant secondary SHPT. When medication fails, surgical intervention is required. Parathyroidectomy (PTX) (2) has been widely adopted as an effective and safe surgery for the treatment of drug-refractory SHPT. It has 3 procedures (2): total parathyroidectomy (TPTX), TPTX + autotransplantation (AT), and subtotal parathyroidectomy (SPTX). Despite their definite short-term efficacy, these 3 procedures will still lead to challenges including persistent hyperparathyroidism (PHPT) or recurrent hyperparathyroidism (RHPT) in the long term. Unlike the risk of the first surgery, reoperation risks can involve patients who have ectopic parathyroid glands, with their associated complex neck adhesions posing a significant challenge to surgeons, in neck operations after the first operation. These complex situations increase the possibility of additional complications in patients undergoing reoperation. Therefore, in order to provide an essential guide to treatment, assessment of the effectiveness and safety of reoperation in SHPT patients is needed. This article summarizes our experience in performing reoperation in 15 patients with drug-refractory SHPT, with an attempt to explore the effectiveness and safety of reoperation. We present the following article in accordance with the STROBE Statement reporting checklist (available at http://dx.doi.org/10.21037/gs-20-391).

\section{Methods}

\section{General data}

The clinical data of 15 patients who underwent reoperation for drug-refractory SHPT in our hospital from August 2010 to March 2019 were retrospectively analyzed. There were 10 females and 5 males aged $(48.00 \pm 8.61)$ (range, $27-$ 62 years). The primary surgeries included SPTX $(n=7)$, TPTX $(n=2)$, TPTX + AT in the forearm $(n=5)$, and TPTX + AT in the sternocleidomastoid muscle $(n=1)$.

\section{Diagnostic criteria for persistent/recurrent SHPT (3)}

PHPT is defined as an intact parathyroid hormone (iPTH) level of greater than $300 \mathrm{pg} / \mathrm{mL}$ present immediately after surgery (3). The definition of RHPT is as follows: iPHT below $300 \mathrm{pg} / \mathrm{mL}$ within 6 months after a successful primary surgery in an SHPT patient but gradually increasing again after 6 months and exceeding $300 \mathrm{pg} / \mathrm{mL}$, accompanied by corresponding clinical symptoms. Overall,
9 patients in our current study met the above criteria for RHPT, and 6 patients met the criteria for PHPT.

\section{Indications for reoperation}

The indications for reoperation included the following (2): (I) iPTH higher than $800 \mathrm{pg} / \mathrm{mL}$; (II) hypercalcemia or hyperphosphatemia unresponsive to drug therapy; (III) parathyroid gland hyperplasia as revealed by ultrasound, gland size diameter $>0.5 \mathrm{~cm}$, and richer blood flow or presence of other imaging evidence; (IV) resistance to medication; and $(\mathrm{V})$ presence of at least 2 of the above criteria plus at least 1 of the following conditions: bone pain, skeletal deformity on imaging, anemia that is difficult to correct, and skin itching.

\section{Pre-operative preparation}

Pre-operative preparation measures included lowphosphorus diet, active vitamin D shock therapy, blood pressure control, erythropoietin therapy, and other conventional treatments. Timing of dialysis for various types of patients was scheduled as follows: 1 session of peritoneal dialysis was performed before surgery on the surgical day, hemodialysis was performed 1 day before surgery, and heparin-free dialysis was performed once. The levels of iPTH and blood biochemical markers were routinely measured before surgery. The cardiopulmonary functions were evaluated by chest digital radiography (DR) or chest computed tomography (CT), cardiac ultrasound, electrocardiography, lung-function testing, and other examinations. Routine examinations performed before surgery included parathyroid ultrasound, 99Tc-MIBI dualtime imaging, neck contrast-enhanced CT scan (plus chest contrast-enhanced CT if necessary), magnetic resonance imaging (MRI) scan of the corresponding sites, and other imaging examinations.

\section{Surgical methods}

For patients with PHPT/RHPT located in the neck or upper mediastinum, general anesthesia was applied. The original neck incision was used as the surgical approach. Intraoperative nerve monitoring was performed to detect and protect the recurrent laryngeal nerve and superior laryngeal nerve. The parathyroid glands were extensively explored during the surgery. The residual parathyroid glands were resected en bloc, and the suspicious parathyroid tissues 
were explored and removed. If the parathyroid glands were located inside the thyroid glands, unilateral thyroidectomy was performed, and the identified parathyroid glands were removed; if the recurrent parathyroid glands were located in the sternocleidomastoid muscle, both the parathyroid glands and the sternocleidomastoid muscle were removed; if the parathyroid glands were located in the upper mediastinum, the upper mediastinum was explored during the operation, and the hyperplasia glands were removed based on the location results received before the operation. Patients undergoing surgical exploration of the neck needed to undergo exploration of the bilateral central areas and resection of the lingual lobe of the thymus. If the recurrence was located in the forearm, the brachial plexus was anesthetized before the parathyroid glands in the forearm and the surrounding muscle tissues were excised according to the preoperative positioning results. Intraoperative frozen-section pathology was performed for all the resected tissues.

\section{Post-operative management}

After the operation, the patient's physical signs were closely monitored, and special attention was paid to complications such as postoperative bleeding, dyspnea, numbness of limbs, convulsions, hoarseness, and choking on water. After routine intravenous calcium $(\mathrm{Ca})$ supplementation and use of high-Ca dialysate, the blood $\mathrm{Ca}$ was monitored, and $\mathrm{Ca}$ gluconate injections $(10 \mathrm{~mL}: 1 \mathrm{~g}) 10-20 \mathrm{~mL}$ bid or tid were administered as appropriate. Oral Ca supplementation was started on the second day after surgery comprising common Ca agent (Ca carbonate) 1-2 $\mathrm{g}$ bid or tid and active vitamin D3 (calciferol) $0.5-1 \mu \mathrm{g}$ bid. The use of intravenous Ca dose was gradually reduced after the fourth day until withdrawal.

\section{Main measures}

The general conditions of the patients were recorded. Changes in main biochemical indicators including $\mathrm{PPTH}$, blood $\mathrm{Ca}$, blood phosphorus (P), alkaline phosphatase (ALP), and blood calcium phosphorus product $\left(\mathrm{Ca}^{*} \mathrm{P}\right)$ before surgery and 1 day, 1 week, 1 month, 3 months, and 6 months after surgery were recorded. The postoperative complications and improvements of clinical symptoms were also recorded. The surgery was regarded as successful if the iPTH level was lower than $136 \mathrm{pg} / \mathrm{mL}$ (2 times the upper limit of the parathyroid glands in our hospital), and an iPTH level of higher than $300 \mathrm{pg} / \mathrm{mL}$ immediately after the operation indicated treatment failure.

\section{Statistical methods}

Statistical analysis was performed by using the SPSS 22.0 software package. The measurement data are presented as $\bar{x} \pm \mathrm{SD}$, and were analyzed with Fisher's exact test. A P value of $<0.05$ was considered statistically significant.

\section{Results}

\section{Surgical results}

The surgically removed parathyroid glands and recurrent glands were consistent with the lesions located by preoperative imaging examinations. As shown in Table 1, these 15 patients underwent reoperation $42.80 \pm 20.84$ months (range, 10-72 months) after the primary surgery, Routine pathological examinations identified a total of 25 parathyroid glands, of which 10 were in the neck in situ, 5 were ectopic in the neck, and 10 were in the forearm. The ectopic parathyroid glands were located inside the thyroid gland ( $\mathrm{n}=1)$, anterior superior mediastinum $(\mathrm{n}=1)$, or thymus $(\mathrm{n}=3)$. In our series, 7 patients experienced recurrence in situ following SPTX, 2 of whom had ectopic parathyroid hyperplasia; 2 patients experienced intrathymic recurrence after TPTX; and 1 patient suffered from recurrence in the upper mediastinum after TPTX + AT in the forearm. Diffuse hyperplasia was confirmed by postoperative pathology in all these patients. In addition, recurrence of the parathyroid glands was found in the forearm in 4 cases and in the sternocleidomastoid muscle in 1 case; postoperative pathology confirmed the presence of nodular hyperplasia in these patients. There were no major postoperative complications such as postoperative hemorrhage or hypocalcemia. In total, 4 patients experienced transient hoarseness and 1 patient suffered from hypothyroidism. No relapse was found during the 6-month follow-up. All patients completed the research and no patients were lost to follow-up (Figure 1). Surgical treatment significantly improved clinical symptoms including skin pruritus and bone pain.

\section{Comparisons of biochemical indicators}

The decrease of iPTH was significantly more remarkable after re-operation than before re-operation $(\mathrm{P}<0.001)$ (Table 2 and Figure 2). During the 6-month follow-up, 
Table 1 Clinical data of 15 patients undergoing reoperation for persistent or recurrent drug-refractory secondary hyperparathyroidism

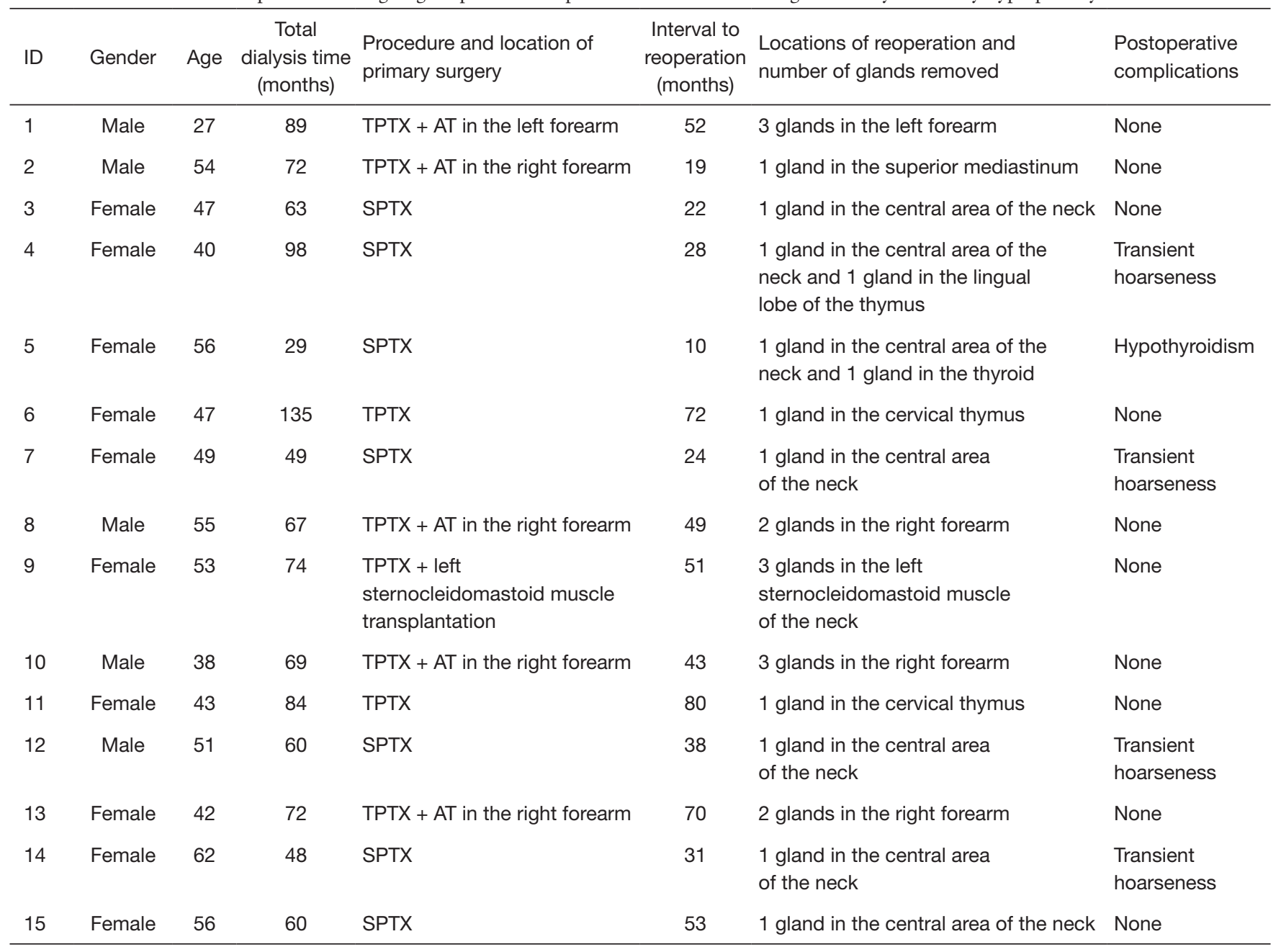

TPTX, total parathyroidectomy; AT, autotransplantation; SPTX, subtotal parathyroidectomy.

iPTH remained stable (about 100), and no recurrence was reported. $\mathrm{Ca}, \mathrm{P}$, and $\mathrm{Ca}^{*} \mathrm{P}$ decreased after reoperation and then slightly increased, showing significant differences compared with the preoperative results $(\mathrm{P}<0.05)$ (Table 2, Figures 3-5). ALP showed a significant downward trend after the reoperation $(\mathrm{P}<0.001)$ (Table 2 and Figure 6).

\section{Discussion}

The production of calcitriol in the kidney gradually decreases in patients with end-stage chronic kidney disease, resulting in disorders of $\mathrm{Ca}$ and phosphorus $(\mathrm{P})$ metabolism along with hyperplasia of parathyroid glands and abnormal production of parathyroid hormone (PTH) (4), which can also include ectopic parathyroid glands. Even after parathyroidectomy, the disorders in $\mathrm{Ca}$ and $\mathrm{P}$ metabolism still exist and may affect extra or ectopic parathyroid glands not found during the primary operation, intentionally preserved parathyroid glands after SPTX, implanted gland fragments after the resected glands are damaged in an accident (5), autotransplanted parathyroid glands, and other parathyroid tissues still inside the human body. All of these postoperative parathyroid tissues can cause PHPT or RHPT. PHPT is characterized by an excessive and unregulated secretion of PTH from 1 or more parathyroid glands; in contrast, RHPT is typified by the progressive proliferation or adenoma degeneration of residual tissues. The interval to progression is shorter when there are more residual glands.

Failed parathyroidectomy is often due to inaccurate positioning of the glands before surgery. Humans usually have 4 parathyroid glands, but they may vary in number 


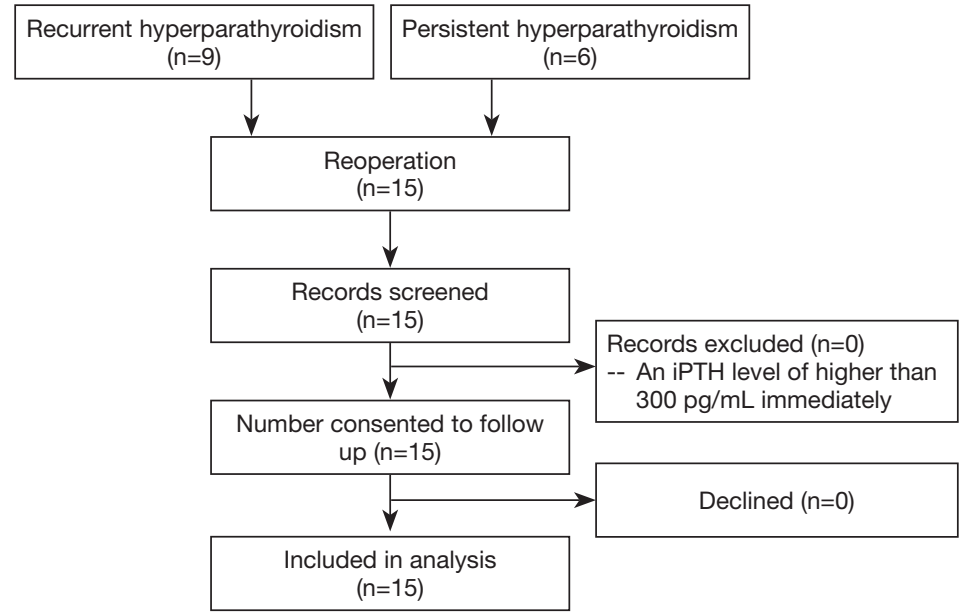

Figure 1 Participants flowchart.

Table 2 Changes of biochemical indicators including iPTH, Ca, P, ALP, and CA*P before and after reoperation for persistent or recurrent drugrefractory secondary hyperparathyroidism

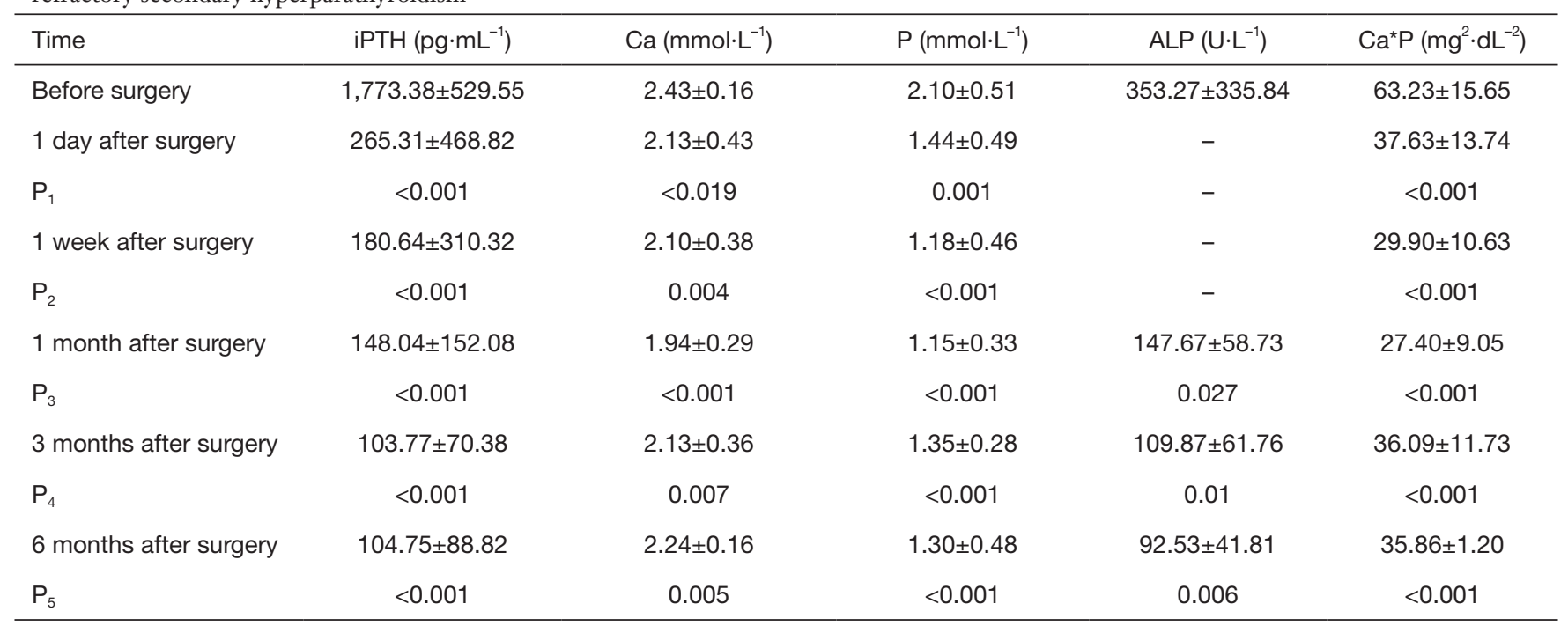

iPTH, intact parathyroid hormone; Ca, blood calcium; P, blood phosphorus; ALP, alkaline phosphatase; Ca*P, blood calcium phosphorus product; $\mathrm{P}_{1}, \mathrm{P}_{2}, \mathrm{P}_{3}, \mathrm{P}_{4}$, and $\mathrm{P}_{5}$, compared with those before reoperation; -, not detected.

and location. During the development of the embryo, the parathyroid glands migrate and separate outward from the third and fourth pharyngeal sacs. However, due to the impact of a variety of factors, ectopic migration (to the sublingual area, lower neck, and even the mediastinum) may occur $(6,7)$, especially for the lower parathyroid glands. Inaccurate preoperative positioning often leads to residual parathyroid glands after the primary operation. Therefore, the primary operation remains a key link for SHPT. The currently available imaging techniques for parathyroid glands include color Doppler ultrasound, dual-time-point $99 \mathrm{mTc}-\mathrm{MIBI}$ imaging, and contrast-enhanced cervical and chest $\mathrm{CT}$ scans or MR scans. Each technique has its own advantages and disadvantages in terms of sensitivity and accuracy. The combinations of various imaging modes are encouraged to improve the accuracy of preoperative positioning, especially for ectopic parathyroid glands. It is a useful strategy to reduce recurrence after surgery (8) and to increase the success rate of surgery.

According to our experience, an operator must carefully 


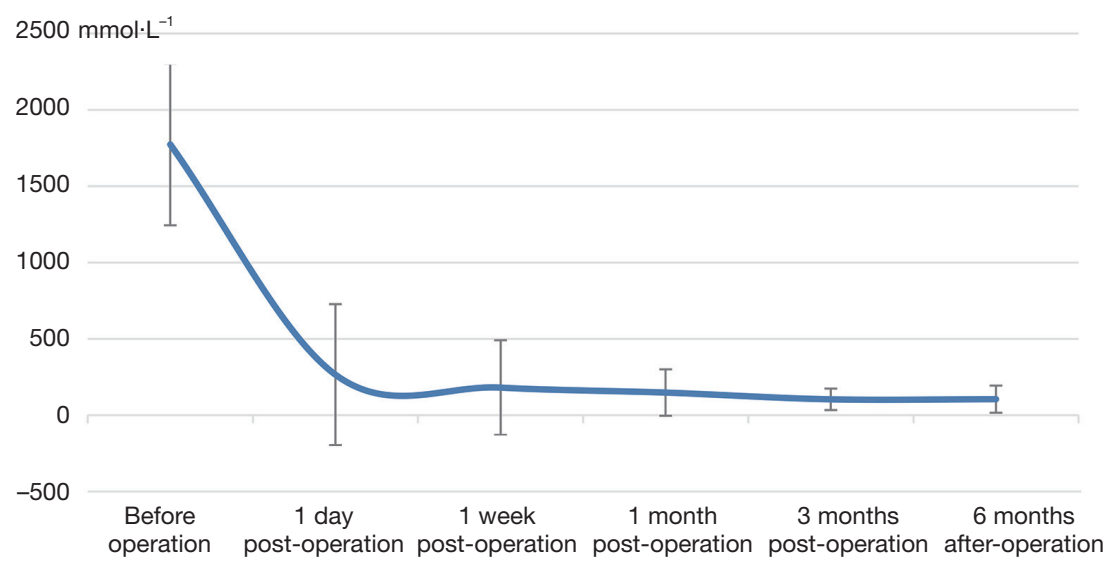

Figure 2 iPTH levels before and after operation. iPTH, intact parathyroid hormone

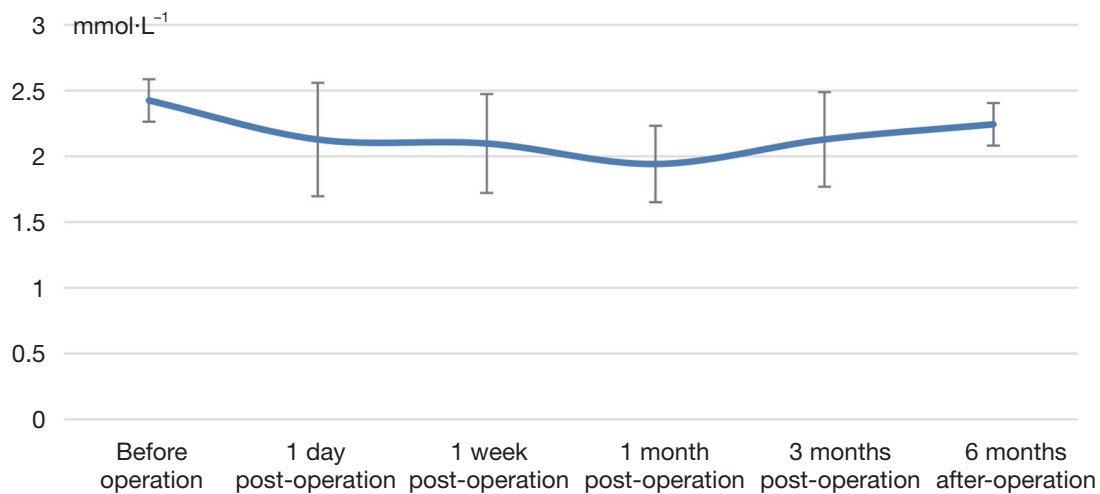

Figure 3 Blood calcium levels before and after operation.

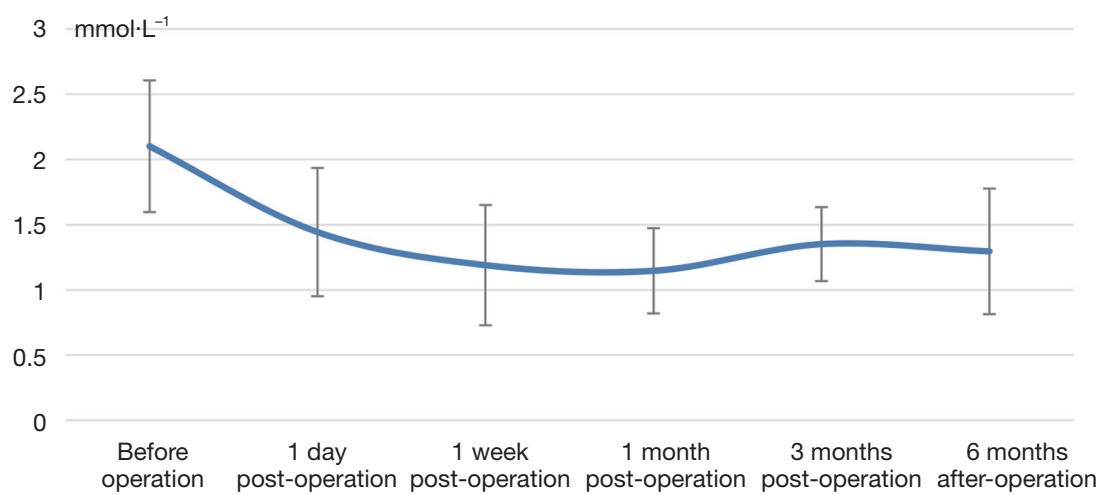

Figure 4 The trend of blood phosphorus before and after operation.

compare the data on a variety of imaging films to accurately locate the parathyroid glands; in particular, the operator may participate in the sonography of parathyroid glands, so as to accurately identify the anatomical locations of the residual parathyroid glands. In our current series, the operators participated in the ultrasound examinations on all the 15 patients, and, based on the ultrasound findings and data from other imaging examinations, they accurately 


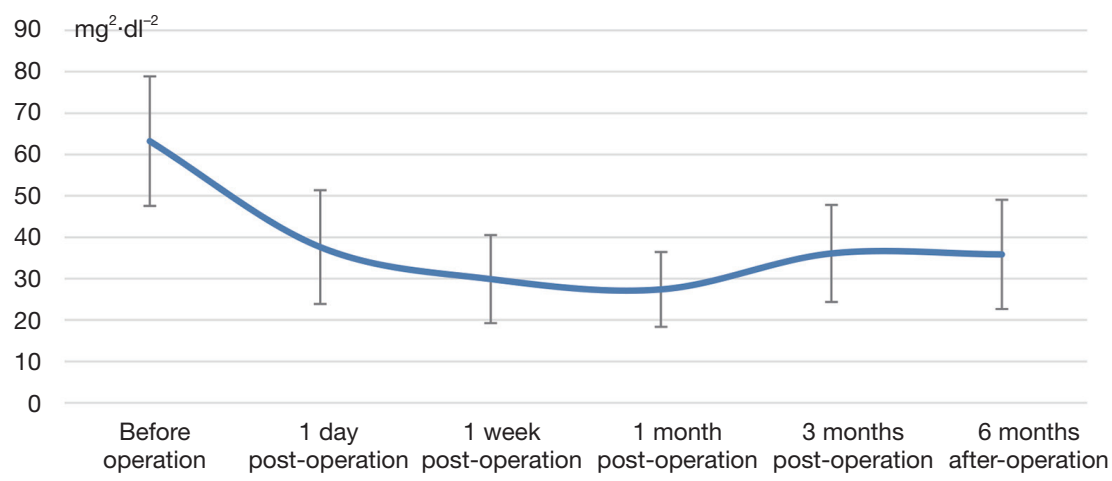

Figure 5 The trend of calcium phosphorus product before and after operation.

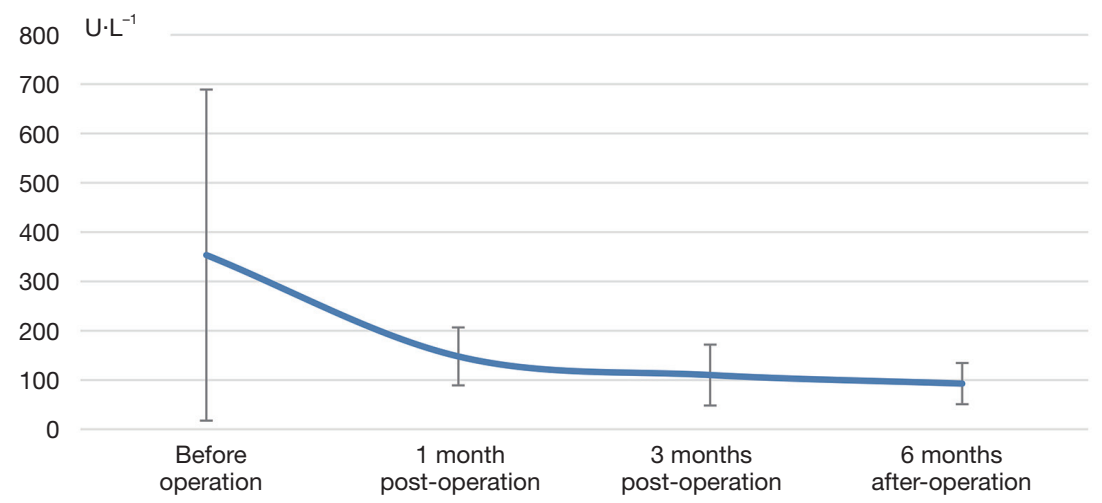

Figure 6 The trend of alkaline phosphatase before and after operation.

located and resected the parathyroid glands.

The choice of 3 surgical procedures for SHPT remains controversial. A meta-analysis by Hou et al. (9) showed that the risk of PHPT or RHPT was highest after SPTX and lowest after TPTX; in addition, the recurrence after TPTX + AT might be explained by excessive parathyroid glands in the forearm. The recurrence in our current series was predominantly caused by residual glands after SPTX. Therefore, a more appropriate surgical and transplantation plan may be another key factor in reducing recurrence.

Severe and untreated SHPT can cause various complications such as calcification, bone marrow fibrosis, and cardiovascular disease $(10,11)$. Surgery is currently the only effective treatment for drug-refractory SHPT. Hou et al. (12) showed that the surgical treatment of SHPT could quickly lower blood iPTH level, relieve itching, bone pain, and other symptoms, and help improve anemia and nutritional status. Therefore, PHPT or RHPT after SHPT also needs to be treated by surgery, and reoperation is also an effective and safe strategy. Special attention should be paid to both the thyroid and thymus during the reoperation, whereas the central area of the neck and the mediastinum should not be ignored (6). Reoperation of the neck is more likely to cause surgical complications due to the dense scar tissue, anatomical distortion, and loss of the tissue planes. Since scar adhesion greatly increases the risk of recurrent laryngeal nerve injury (13), the nerves should be carefully protected during the reoperation (2). Meanwhile, for the recurrence in the forearm after SHPT, surgery is simpler and safer. It can be performed under local anesthesia or cervical plexus anesthesia and avoids the complications caused by the neck exploration during reoperation, which greatly improves patient safety.

In summary, reoperation is an effective and safe treatment for drug-refractory SHPT and can be actively applied in clinical settings. When reoperation is planned, multiple imaging studies should be performed to accurately locate ectopic or hyperplastic parathyroid glands. All the possible parathyroid glands must be removed to prevent any recurrence. 


\section{Acknowledgments}

Funding: Lishui Municipal Science and Technology Program (2019SJZC44).

\section{Footnote}

Reporting Checklist: The authors have completed the STROBE Statement reporting checklist. Available at: http://dx.doi.org/10.21037/gs-20-391.

Data Sharing Statement: Available at http://dx.doi. org/10.21037/gs-20-391.

Conflicts of Interest: All authors have completed the ICMJE uniform disclosure form (available at http://dx.doi. org/10.21037/gs-20-391). All author report grants from Lishui Science and Technology Bureau, during the conduct of the study.

Ethical Statement: The authors are accountable for all aspects of the work in ensuring that questions related to the accuracy or integrity of any part of the work are appropriately investigated and resolved. The research protocol was reviewed and approved by the Clinical Research Medical Ethics Committee of Lishui Central Hospital, China (no. 2018-82).

Open Access Statement: This is an Open Access article distributed in accordance with the Creative Commons Attribution-NonCommercial-NoDerivs 4.0 International License (CC BY-NC-ND 4.0), which permits the noncommercial replication and distribution of the article with the strict proviso that no changes or edits are made and the original work is properly cited (including links to both the formal publication through the relevant DOI and the license). See: https://creativecommons.org/licenses/by-nc-nd/4.0/.

\section{References}

1. Ni LH, Yuan C, Song KY, et al. Efficacy and safety of cinacalcet and active vitamin $\mathrm{D}$ in the treatment of secondary hyperparathyroidism in patients with chronic kidney disease: a network meta-analysis. Ann Transl Med 2019;7:322.

2. Tian W, He QQ, Jiang KW, et al. Expert Consensus on the Clinical Practice of Surgical Treatment for Secondary
Hyperparathyroidism in Patients with Chronic Renal

Failure. China Journal of Practical Surgery 2016;36:481-6.

3. Oltmann SC, Madkhali TM, Sippel RS, et al. Kidney Disease Improving Global Outcomes guidelines and parathyroidectomy for renal hyperparathyroidism. J Surg Res 2015;199:115-20.

4. Jamal SA, Miller PD. Secondary and tertiary hyperparathyroidism. J Clin Densitom 2013;16:64-8.

5. Abruzzo A, Gioviale MC, Damiano G, et al. Reoperation for persistent or recurrent secondary hyperparathyroidism. Surgical treatment of renal hyperparathyroidism. Acta Biomed 2017;88:325-8.

6. Theurer S, Siebolts U, Lorenz K, et al. Ectopic tissue of the thyroid gland and the parathyroid glands. Pathologe 2018;39:379-89.

7. Kowa XY, Richards P, Waterhouse M, et al. Atypical presentations of parathyroid gland pathology: A pictorial review. Eur J Radiol Open 2019;6:320-9.

8. Xu D, Yin Y, Hou L, et al. Surgical management of secondary hyperparathyroidism: how to effectively reduce recurrence at the time of primary surgery. J Endocrinol Invest 2016;39:509-14.

9. Hou J, Shan H, Zhang Y, et al. Network meta-analysis of surgical treatment for secondary hyperparathyroidism. Am J Otolaryngol 2020;41:102370.

10. Harmacek D, Arampatzis S. Arteriosklerosis and "osteoporosis" in patients with chronic kidney disease: same same, but different! Ther Umsch 2018;75:371-5.

11. Tentori F, Wang M, Bieber BA, et al. Recent changes in therapeutic approaches and association with outcomes among patients with secondary hyperparathyroidism on chronic hemodialysis: the DOPPS study. Clin J Am Soc Nephrol 2015;10:98-109.

12. Hou AZ, Xiao GQ, Kong YZ, et al. Clinical effect of total parathyroidectomy combined with forearm autograft in uremic patients complicated with secondary hyperparathyroidism. Chinese Journal of Blood Purification 2016;15:285-8.

13. Wojtczak B, Barczyński M. Intermittent neural monitoring of the recurrent laryngeal nerve in surgery for recurrent goiter. Gland Surg 2016;5:481-9.

Cite this article as: Zhu L, Cheng F, Zhu X, Zhou B, Xu Y, Wu Y, Shao C. Treatment of persistent or recurrent drugrefractory secondary hyperparathyroidism with reoperation. Gland Surg 2020;9(2):401-408. doi: 10.21037/gs-20-391 